\title{
Correlation of Self and Solute Diffusion Data with Cohesive Energy
}

\author{
By R. V. Patil* and G. P. Tiwari*
}

\begin{abstract}
The paper discusses the correlation of the diffusion data with cohesive energy. The free energy for self diffusion of metals at the melting point is shown to be a linear function of their cohesive energies. In the case of solute diffusion, it is shown that the excess free energy for impurity-diffusion at the melting point is similarly related to the partical molar free energy of the solute atom as determined on the basis of the Hildebrand's regular solution model.
\end{abstract}

(Received February 25, 1974)

\section{Introduction}

The empirical correlations of the diffusion parameters with the physical properties are useful in the discussion of diffusion behaviour. They serve two main purposes. First, they help in making an order of magnitude estimation of diffusion data, where experimental measurements are not available. Secondly, they are also useful in establishing the mode of diffusion. Any radical departure from the empirical correlation is an indication of the change in the diffusion mechanism. The correlations also provide important insight into the process of diffusion. The relationship observed between the melting point and the activation energy ${ }^{(1)}$ has, for instance, led to the concept that the melting of the solid occurs when a fixed fraction of the lattice sites are vacant. In a similar manner, Toth and Searcy ${ }^{(2)}$ have correlated the activation energy for vacancy migration with the compressibility. It is based on the hypothesis that the atoms in the saddle-point configuration experience compressive stresses.

In the present paper, a correlation is shown to exist between the self and solute diffusion data and the cohesive energy of the solid. Cohesive energy is a measure of the strength of the bonding. Self and substitutional diffusion in crystalline solids can be visualised as the breaking of the atomic bonds near a vacancy (where half of the bonds among the nearest neighbours are broken already) and their reformation at the next site. It appears intuitively logical that the energy associated with the process of diffusion should be related to the cohesive energy of the solid.

\section{Self-diffusion}

The essential steps in the vacancy controlled selfdiffusion in metals are the formation and migration of the vacancies. The formation of vacancy is equivalent to breaking of ' $Z$ ' bonds inside a crystal and restoring $\frac{1}{2} \mathrm{Z}$ bonds at the surface, where $\mathrm{Z}$ is the coordination number. Hence the energy involved in formation of a vacancy can be approximated to the strength of $\frac{1}{2} Z$ bonds in the crystal ${ }^{(3)}$. This is an upper limit to the

* Metallurgy Division, Bhabha Atomic Research Centre, Trombay, Bombay-400085, India

Trans. JIM vacancy formation energy, since the relaxation of atoms around a vacancy is not considered. Again, at the saddle-point, the diffusing atom is hemmed in from all sides by its nearest neighbours. The distance between the atoms involved in the saddle-point configuration is less than the equilibrium distance leading to distortion of the atomic bonds. Thus, the energy formation as well as migration of vacancies, is depend-

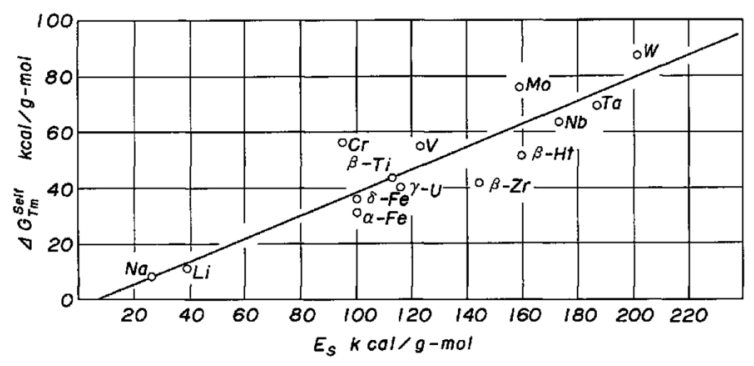

Fig. 1 A plot of free energy for self-diffusion at the melting point versus cohesive energy for $b c c$ metals.

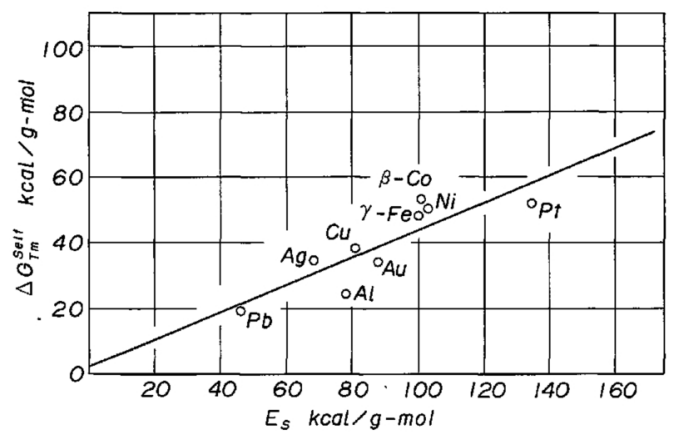

Fig. 2 A plot of free energy for self-diffusion at the m.p. versus cohesive energy for $f c c$ metals.

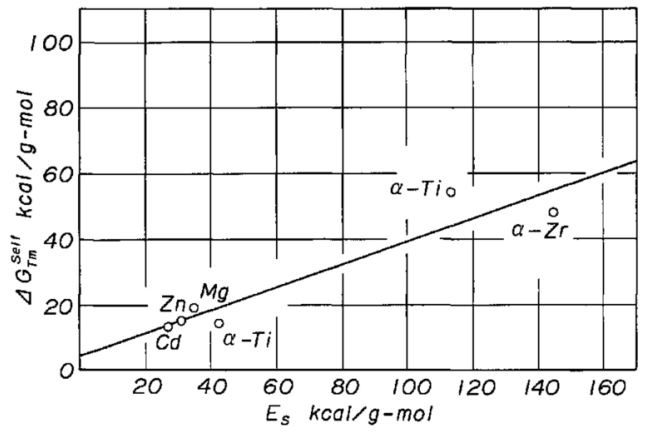

Fig. 3 A plot for free energy for self diffusion at m.p. versus cohesive energy for $h c p$ metals.

1974 Vol. 15 
ent upon the strength of atomic bond. Since the cohesive energy of a crystal is derived from these bonds, the cohesive strength of a crystal should be related to the energy involved in the process of diffusion. Further, the constant of proportionality between these quantities should be a function of the crystal structure.

In Figs. 1-3, free energies for self-diffusion at the melting point are plotted against the cohesive energies for the common $f c c, b c c$ and $h c p$ metals. According to Nachtrieb et al. ${ }^{(4)}$ the substances must be in the corresponding states for the purpose of comparing diffusion behaviour. The melting point has been shown to be the ideal reference state as the change in meltingpoint due to the application of pressure or alloying does not affect the diffusion rates at the melting temperature. Instead of activation energy, the free energy for diffusion has been plotted against the cohesive energy as the latter quantity takes into account the variation in the frequency factor also. The equations representing the relation between the cohesive energy $\left(E_{s}\right)$ and the free energy for self diffusion at the melting point $\left(\Delta G_{T m}^{\text {Self }}\right)$

$$
\begin{array}{ll}
b c c & \Delta G_{T m}^{\text {Self }}=0.39 E_{s}-1.73, \\
f c c & \Delta G_{T m}^{\text {Self }}=0.41 E_{s}+1.95, \\
h c p & \Delta G_{T m}^{\text {Self }}=0.35 E_{s}+4.00,
\end{array}
$$

while the data for the Gibbs free energy for self diffusion have been taken from Gibbs' paper ${ }^{(5)}$, the cohesive energy values are from Kepart's book on "Early Transition Metals"(6). The coefficient of correlation of the data points for the eqs. (1), (2) and (3) are $0.90,0.87$ and 0.95 respectively. The present correlation covers normal as well as anomalous diffusion behaving metals such as $\beta-\mathrm{Zr}, \beta-\mathrm{Ti}, \gamma-\mathrm{U}$, etc. Hence, it can be seen from Figs. 1-3, that the anomalies found in the application of melting point rule are not observed with this correlation.

\section{Impurity Diffusion}

The impurity diffusion of a solute element in a solvent matrix is controlled by its 'in solution' charge and size. In solution charge and the size of the solute is a function of its partial molar free energy. It is therefore reasonable to expect that the solute diffusion characteristics in a particular solvent should exhibit a simple relationship to their partial molar free energies. Hildebrand and $\operatorname{Scott}^{(7)}$ have shown that the partial molar free energy of a solute $\left(\Delta G_{i}\right)$ can be expressed as

$$
\Delta G_{i}=R \ln x_{i}+V_{i}\left[\left(\frac{E_{s}}{\Omega}\right)_{i}^{1 / 2}-\left(\frac{E_{s}}{\Omega}\right)_{0}^{1 / 2}\right]^{2} \phi_{0}^{2},
$$

where $x_{i}, V_{i}$ are the mole fraction and the molar volume of the solute, $\left(E_{s} / \Omega\right)_{i}$ and $\left(E_{s} / \Omega\right)_{0}$ are the ratios of the cohesive energy to the atomic volume $(\Omega)$ for the solute and solvent respectively, and $\phi_{0}$ is the volume fraction of the solvent.

The requirements of a regular solution, viz. no

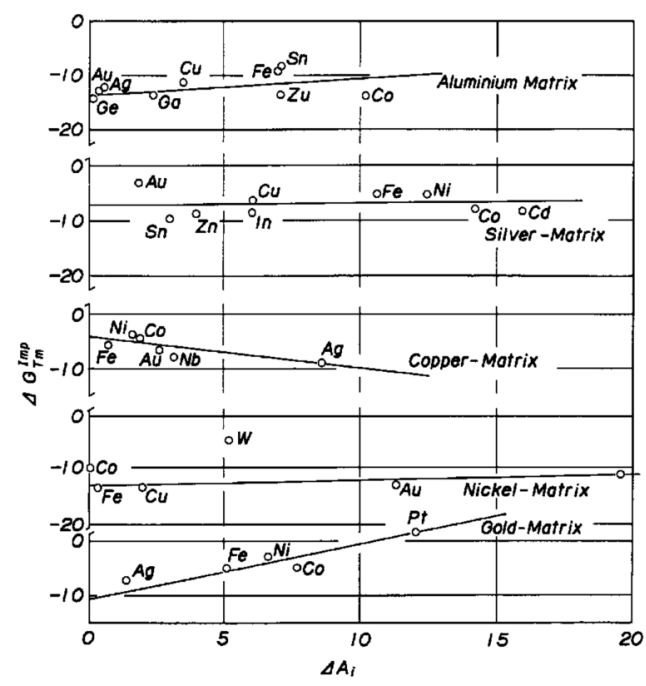

Fig. 4 A correlation of the excess free energy for solute diffusion at melting point vs partial molar free energy of solute for impurity diffusion in metals.

change in the entropy of mixing and a negligible change in the volume, are met in the radioactive tracer diffusion due to an extremely small amount of solute involved in the process. Further, the quantities $x$ and $\phi$ do not vary with the solute and therefore, any change in the partial molar free energies of different solutes is due to the last term $V_{i}\left[\left(E_{s} / \Omega\right)_{i}^{1 / 2}-\left(E_{s} / \Omega\right)_{0}^{1 / 2}\right]^{2}$ in eq. (4).

In the Fig. 4, the change in the free energy for impurity diffusion at the melting point of the matrix $\left(\Delta G_{T m}^{\mathrm{imp}}\right.$. $)$ has been plotted against $\Delta A_{i}\left\{=V_{i}\left[\left(E_{s} / \Omega\right)_{i}^{1 / 2}\right.\right.$ $\left.\left.-\left(E_{s} / \Omega\right)_{0}^{1 / 2}\right]^{2}\right\}$ for some $f c c$ matrices. The impurity diffusion data used for calculating $\Delta G_{T m}^{\mathrm{imp}}$, is taken from the Askill Report ${ }^{(8)}$. In plotting Fig. 4, it is assumed that the free energy of the solute at the equilibrium point changes by a constant fraction when it moves to the saddle point. Further, this fraction is same for all solutes diffusing through a matrix by the identical mechanism. It is seen from Fig. 4 that the impurity diffusion data for all solvents lie in the straight lines. This linear relationship between $\Delta G_{T m}^{\operatorname{imp}}$. and $\Delta A_{i}$ confirms the above-mentioned assumption for vacancy controlled diffusion in metals.

The above correlation provides a simple way of rationalising impurity diffusion data. For instance the diffusion of transition metals in aluminium ${ }^{(9)}$ yields very large entropy factors and high activation energy values. The activation energies have been quantitatively explained on the basis of screening theory. However, no explanations have been advanced for the large magnitude of entropy factor. This anomaly ceases to exist if instead of activation energies, free energies of diffusion, as in the present correlation, are employed for comparing the diffusion characteristics of the solutes.

\section{Acknowledgments}

Our thanks are due to Dr. B. D. Sharma and Mr. V. S. Raghunathan for many helpful discussions. 
They also appreciate the interest of Dr. M. K. Asundi, Head, Physical Metallurgy Section in this work. Permission to publish this paper from Head, Metallurgy Division, is gratefully acknowledged.

\section{REFERENCES}

(1) G. V. Kidson and R. Ross: Radioisotopes in Scientific Research, Pergamon Press, New York, (1958), p. 185.

(2) L. E. Toth and A. W. Searcy: University of California Re. UCRL-10581 (1963).
(3) M. W. Thompson: Defects and Radiation Damage in Metals, Cambridge University Press, (1969), p. 11.

(4) H. A. Resing and N. H. Nachtrieb: J. Phys. Chem. Solids, 21 (1961), 40.

(5) G. B. Gibbs: Acta Met., 12 (1964), 674.

(6) D. L. Kepert: The Early Transition Metals, Academy Press, London, New York (1972), p. 34.

(7) J. H. Hildebrand and R. L. Scott: Solubility of Nonelectrolytes, Dover Publications Inc., New York (1964), p. 134.

(8) J. Askill: A Bibliography on Tracer Diffusion in Metals, USAEC Rep. ORNL-3795, (1965).

(9) G. M.Hood and R. J. Schultz: Phil. Mag., 23 (1971), 1419. 